\title{
O paradoxo de Simpson: um estudo de caso demográfico sobre dinâmica da população, pobreza e desigualdade
}

\author{
Simpson's paradox: a demographic case study of population \\ dynamics, poverty, and inequality
}

Raphael Mendonça Guimarães (https://orcid.org/0000-0003-1225-6719) 1,2

Flávia Cristina Drumond Andrade (https://orcid.org/0000-0002-3856-3816) ${ }^{2}$
${ }^{1}$ Escola Politécnica de Saúde Joaquim Venâncio, Fundação Oswaldo Cruz. Avenida Brasil 4365 Sala 314, Manguinhos. 21041360 Rio de Janeiro RJ Brasil.raphael.guimaraes@ fiocruz.br

${ }^{2}$ University of Illinois at Urbana Champaign. Illinois Estados Unidos.

\begin{abstract}
Brazil is undergoing a demographic transition characterized by regional inequalities. It is reasonable to assume that aspects related to poverty, development and inequality might reverse the sign of the association of indicators of demographic transition, exemplifying a phenomenon known as Simpson's Paradox. The aim of this study was to analyze the effect of inequality, poverty and social development on population dynamics in Brazil, verifying the occurrence of Simpson's paradox in demographic transition. We used population data from the 1991, 2000 and 2010 national censuses, broken down by age and federative unit (FU). The correlation between demographic indicators was assessed by stratifying the FUs into groups according to their median social indicators. The findings show that all FUs have progressed against social indicators and are undergoing demographic transition; however, despite reductions in disparities over the study period, persistent gaps exist between regions. Simpson's paradox was present when the analysis was carried out by census year and social indicators, and was particularly pronounced in 1991. The main challenge is to define how to analyze demographic dynamics in Brazil and understand how contextual factors alter the pace, quantum, and pattern of demographic transition.
\end{abstract}

Key words Demography, Demographic transition, Poverty, Inequality, Development
Resumo O Brasil experimenta uma transição demográfica marcada por desigualdades regionais. É possível supor que aspectos relacionados à pobreza, desenvolvimento e desigualdade possam reverter os efeitos de associação dos indicadores da transição demográfica, tipificando um fenômeno conhecido como Paradoxo de Simpson. O objetivo foi analisar o efeito da desigualdade, pobreza e desenvolvimento social na dinâmica populacional brasileira, verificando a ocorrência do paradoxo de Simpson na transição demográfica. Foram utilizados dados populacionais oriundos dos Censos Demográficos brasileiros de 1991 a 2010, segundo idade e unidades da federação. Foi avaliada a correlação entre os indicadores demográficos, estratificando das unidades da federação em grupos de acordo com os indicadores sociais. Há um avanço das unidades federativas (UF) com relação aos indicadores sociais. A transição vem ocorrendo em todas as UF, com persistência da distância entre elas, mesmo que com redução ao longo dos anos. Observou-se o paradoxo de Simpson quando a análise foi realizada segundo ano censitário $e$ indicador social, principalmente para o ano de 1991. O principal desafio é compreender como a dinâmica demográfica brasileira pode ser analisada e compreender de que forma os fatores contextuais alteram seu ritmo, quantum e padrão.

Palavras-chave Demografia, Transição demográfica, Pobreza, Iniquidade, Desenvolvimento 


\section{Introdução}

A Transição Demográfica é um modelo que descreve a mudança populacional ao longo do tempo. Elaborada em 1929 pelo demógrafo americano Warren Thompson, é baseado em uma interpretação das mudanças observadas, ou transições, nas taxas de nascimento e morte nas sociedades industrializadas nos últimos duzentos anos que a antecederam ${ }^{1}$. Segundo esta teoria, houve, inicialmente, uma queda nas taxas de mortalidade, com a sustentação da natalidade, num primeiro momento. Em seguida, há um balanço positivo no crescimento vegetativo, levando a um aumento no volume populacional ${ }^{2}$.

Com relação aos nascimentos, a análise pioneira de Phillipe Ariès sobre a história da infância, e as motivações e constrangimentos para a fecundidade desencadeiam um conjunto de teorias microeconômicas, seguida de teorias culturais que explicam as razões para a queda da fecundidade, relacionando-a ao custo dos filhos, à qualificação material destes filhos e, finalmente, no sentido de autorrealização na vida adulta, em que a parentalidade é, em alguma medida, substituída por outros componentes do estilo de vida ${ }^{3}$. Para os óbitos, em contrapartida, há um conjunto de teorias que descrevem o comportamento da mortalidade associada especialmente aos aspectos da determinação social do processo saúde doença ${ }^{4}$, e da desigualdade como causa fundamental dos mecanismos de adoecimento da população ${ }^{5}$. Desta forma, viu-se emergir diversas teorias sociais distintas daquela usada rotineiramente para explicar o processo de dinâmica demográfica, reduzida ao comportamento quantitativo de nascimentos, óbitos e crescimento de produção ${ }^{6}$.

A primeira ou "clássica" transição demográfica refere-se ao declínio histórico na mortalidade e natalidade, cujo encerramento seria a obtenção de uma população estacionária e mais envelhecida, com uma fecundidade de reposição, crescimento populacional zero e esperança de vida superior a $70 \mathrm{anos}^{7}$. Naquele contexto, considerava-se haver um equilíbrio entre mortes e nascimentos, e não era necessária uma migração sustentada para manter o volume populacional. Além disso, cabe ressaltar que, naquele momento da história, havia um padrão familiar nuclear e conjugal ${ }^{8}$.

A maioria das regiões e países vem experimentando, nos últimos 200 anos, mudanças demográficas sem precedentes. Contudo, as projeções apontam que tais mudanças tendem a tornar os países com perfis bastante divergentes, com estagnação ou declínio potencial em partes do mundo desenvolvido e crescimento rápido continuado nas regiões menos desenvolvidas. Portanto, as sociedades contemporâneas estão agora em estágios muito diferentes de suas transições demográficas9. Ou seja, embora a transição tenha ocorrido com padrão relativamente homogêneo nos países industrializados, a teoria e o modelo são frequentemente imprecisos quando aplicados a países de economia periférica devido a fatores sociais, políticos e econômicos específicos que afetam populações específicas ${ }^{10}$.

Com a maior população da América Latina, e maior extensão territorial, o Brasil está passando por este processo. Atualmente, a maior parte da população vive em áreas urbanas e vem experimentando um declínio mais acelerado da fecundidade, desde a década de 1970, um movimento posterior à queda da mortalidade, iniciado ainda nos anos de $1930^{11}$. Aliado ao seu tamanho continental, o país possui marcada desigualdade regional. Embora o Brasil tenha reduzido as desigualdades a partir da base, ou seja, retirando parte da população brasileira localizada abaixo da linha de pobreza, a grande concentração de renda no topo se manteve estável ${ }^{12}$. Cabe ressaltar que o atual contexto nacional joga contra esses necessários avanços, com a acentuação da crise fiscal, a partir de 2014, e as medidas de austeridade fiscal adotadas a partir do golpe de $2016^{13}$.

Evidentemente, por se tratar de um modelo, representa uma generalização que conta com relativa imprecisão na descrição dos casos individuais. Neste sentido, há não apenas teorias sociais e econômicas que procuram detalhá-la melhor, como uma diversidade de modelos operacionais que diferem em técnicas de estimação das componentes demográficas e do peso atribuído a elas para explicar o fenômeno da transição em $\mathrm{si}^{14}$. Entretanto, poucos estudos exploram variáveis que possam em parte explicar o efeito da transição, tanto no padrão quanto no nível com que as mudanças ocorrem. A este respeito, é possível supor que aspectos relacionados à pobreza, desenvolvimento e desigualdade social possam representar confundidores que revertem o sinal da associação destas características, seja por ter relação direta com o padrão de nascimentos, seja com a mudança na probabilidade de morte ${ }^{15-19}$. Esta situação tipifica um fenômeno conhecido como Paradoxo de Simpson.

O paradoxo de Simpson é uma condição extrema de confusão em que uma associação aparente entre duas variáveis é revertida quando os 
dados são analisados dentro de cada estrato de uma variável de confusão. Com o paradoxo de Simpson, a correlação marginal entre causa e efeito seria considerada espúria, ou seja, pode ser inferida como causal porque um terceiro fator funciona como causa da correlação entre as variáveis. Este efeito pode levar a uma conclusão equivocada de que uma determinada associação é verdadeira, quando na verdade não é ${ }^{20}$. Para que esse paradoxo ocorra, duas condições devem estar presentes: (a) uma variável de confusão ignorada ou negligenciada que tem um forte efeito na variável de resultado; e (b) uma distribuição desproporcional da variável de confusão entre os grupos sendo comparados ${ }^{21}$. Este fenômeno há muito foi reconhecido como uma possibilidade teórica, mas poucos exemplos reais foram apresentados. Diante disso, o objetivo do presente estudo é analisar o efeito da pobreza e desigualdade na dinâmica populacional brasileira, verificando a ocorrência do paradoxo de Simpson na transição demográfica.

\section{Materiais e método}

\section{Contexto}

Trata-se de um estudo ecológico, cuja unidades de análise são as unidades federativas (UF), num total de 27, e que são entidades subnacionais com certo grau de autonomia (autogoverno, auto legislação e auto arrecadação) e dotadas de governo e constituição próprios, que unidas formam a República Federativa do Brasil ${ }^{22}$. Elas são constituídas por 26 estados e um distrito federal, localizados em cinco grandes regiões. De forma semelhante ao que ocorreu em muitos países de todos os continentes, os condicionantes dos processos de regionalização, que respondem por transformações territoriais no Brasil obedecem a critérios demográficos, econômicos, políticos e sociais. É importante ressaltar, contudo, que este processo é igualmente marcado pelo incremento das desigualdades entre classes sociais e territórios. Desta forma, estas unidades federativas, a despeito do progresso brasileiro nas áreas social e econômica, mantém bastante heterogeneidade com relação aos indicadores de pobreza, desigualdade, renda e desenvolvimento social ${ }^{23}$.

\section{Fonte de Dados}

Foram utilizados dados populacionais oriundos dos Censos Demográficos brasileiros de 1991,
2000 e 2010, levantados pela Fundação Instituto Brasileiro de Geografia e Estatística (IBGE), segundo idade no Brasil, desagregados por UF. Adicionalmente, foram extraídos os seguintes indicadores sociais do Atlas do Desenvolvimento Humano do Brasil24:

a) Índice de Desenvolvimento Humano (IDH): é uma medida resumida do progresso a longo prazo em três dimensões básicas do desenvolvimento humano: renda, educação e saúde. A mais recente metodologia de cálculo do IDH considera: a expectativa de vida como medida proxy de uma vida longa e saudável (saúde); o padrão de vida (renda), medido pela Renda Nacional Bruta (RNB) per capita expressa em poder de paridade de compra (PPP) constante, em dólar, tendo 2005 como ano de referência; e o acesso ao conhecimento (educação), medido por: i) média de anos de educação de adultos, que é o número médio de anos de educação recebidos durante a vida por pessoas a partir de 25 anos; e ii) a expectativa de anos de escolaridade para crianças na idade de iniciar a vida escolar. Para o seu cálculo são considerados os índices de cada dimensão, estimados por

$\underset{\text { Dimensão }}{\text { Índice da }}=\frac{(\text { valor observado }- \text { valor mínimo })}{(\text { valor máximo }- \text { valor mínimo })}$

Os índices então são calculados para as seguintes dimensões: Expectativa de vida ao nascer (EV), Índice de Educação (IE) (obtido através das subdimensões "Índice de Anos Médios de Estudo" (IAME) e "Índice de Anos Esperados de Escolaridade" (IAEE)); e Índice de Renda (IR). A medida síntese é obtida a partir da média geométrica dos três índices anteriores normalizados:

$$
\mathrm{IDH}=\sqrt[8]{\mathrm{EV} \times \mathrm{IE} \times \mathrm{IR}}
$$

b) Coeficiente de Gini: é uma medida de desigualdade que avalia a concentração de renda numa população. O coeficiente de Gini é utilizado para medir a desigualdade de distribuição de renda. Ele varia de 0,0 (igualdade perfeita, com todos os ganhos das famílias exatamente o mesmo) a 1,0 (desigualdade absoluta, com uma única casa ganhando renda inteira de uma localidade). Matematicamente, o coeficiente de Gini é equivalente a metade da diferença média absoluta entre os rendimentos de quaisquer dois domicílios amostrados aleatoriamente de uma população, e em seguida, normalizados para a média. $O$ coeficiente de Gini pode ser calculado com a Fórmula de Brown:

$$
\mathrm{G}=1-\sum_{\mathrm{k}=0}^{\mathrm{k}=\mathrm{n}-1}\left(\mathrm{X}_{\mathrm{k}+1}-\mathrm{X}_{\mathrm{k}}\right)\left(\mathrm{Y}_{\mathrm{k}+1}+\mathrm{Y}_{\mathrm{k}}\right)
$$


onde: $\mathrm{G}$ = coeficiente de Gini; $\mathrm{X}=$ população; $\mathrm{Y}=$ renda

c) Proporção de extremamente pobres: Proporção dos indivíduos com renda domiciliar per capita igual ou inferior a 1/4 do salário-mínimo.

d) Renda média per capita: renda média de cada residente de determinada UF. Trata-se da a soma da renda de todos os residentes, dividida pelo número de habitantes.

Os dados populacionais permitiram o cálculo dos seguintes indicadores demográficos:

i) Taxa Bruta de Natalidade (TBN): número de nascidos vivos, por mil habitantes;

ii) Taxa Bruta de Mortalidade (TBM): número total de óbitos, por mil habitantes.

\section{Análise de Dados}

O Brasil possui uma cobertura incompleta dos nascimentos e mortes nos registros vitais ${ }^{25}$. Por esta razão, os indicadores de natalidade e mortalidade frequentemente são obtidos através de dados censitários e pesquisas retrospectivas padronizadas, como a Pesquisa Demográfica de Saúde. Dessa forma, é necessário efetuar o ajuste dos dados com a utilização de métodos demográficos indiretos. Nesta pesquisa, utilizados os dados censitários dos anos de 1991, 2000 e 2010, e corrigimos a subcontagem de nascimentos no registro de nascimento no Brasil usando os fatores de ajuste fornecidos pelo Instituto Brasileiro de Geografia e Estatística, através de métodos estabelecidos de ajuste de dados, como o método relacional P/F de Brass e Gompertz ${ }^{26}$. Para a correção dos dados de mortalidade, utilizamos: o método Adjusted Synthetic Extinct Generations (SEG-adj), proposto por Hill et al. ${ }^{27}$, para a mortalidade adulta; e para a mortalidade infantil utilizamos o método de Brass e Coale ${ }^{28}$ com a variante proposta por Trusell ${ }^{29}$.

Os indicadores sociais foram ranqueados para que fosse possível avaliar a heterogeneidade das unidades brasileiras com relação à desigualdade, pobreza e desenvolvimento social. Em seguida foram comparados os indicadores por UF nos anos censitários e, em seguida, estimada a sua variação ao longo dos 20 anos da série temporal estudada. Para verificar se há o efeito de tempo na relação entre natalidade e mortalidade - o que é indicativo da transição demográfica -, procedemos ao cálculo da correlação entre estas duas taxas para cada ano censitário e comparamos a direção da correlação e sua magnitude. Adicionalmente, foi estimada a correlação entre os indicadores demográficos (natalidade e mortalida- de) e os indicadores econômicos (Índice de Gini, renda média per capita, proporção de extremamente pobres e IDH), para cada ano. Ainda, nós aplicamos os valores de referência do Brasil em 1991 para observar a mudança das UF com seus dados de 1991, 2000 e 2010. A fim de suavizar a correlação, quando ela não foi linear, foram utilizadas análises do tipo lowess (locally weighted running line smoother) para a avaliação das relações entre as variáveis de interesse.

A partir deste diagnóstico inicial, as UF foram divididas em dois grupos, a partir da mediana dos indicadores sociais, e os indicadores demográficos foram então avaliados a partir da estratificação dos dois grupos. Uma vez que, conceitualmente, a transição demográfica é marcada por uma mudança nos padrões de natalidade e mortalidade, sintetizamos a relação entre as taxas brutas de natalidade e mortalidade. As medidas de correlação foram feitas através do coeficiente de Pearson. Foi medida ainda a significância estatística das medidas calculadas.

\section{Resultados}

Há um avanço geral dos estados brasileiros com relação aos indicadores sociais. A Figura 1 apresenta o ranking das UF para os anos 1991, $2000 \mathrm{e}$ 2010. A Figura 2 apresenta a variação relativa dos indicadores entre os anos de 1991 e 2010 (para inspeção visual da mudança destes indicadores nos três anos censitários, vide Tabela 1, Figuras 3 e 4). A evolução do Índice de Desenvolvimento Humano (IDH) evidencia crescimento global do desenvolvimento humano nas unidades federativas, com redução da desigualdade. Importante destacar que há uma certa formação de aglomerados espaciais de IDH semelhantes. Nós consideramos as faixas de IDH propostas pela Organização das Nações Unidas ${ }^{17}$ (IDH muito baixo até 0,444 ; baixo entre 0,500 e 0,599 ; médio entre 0,600 e 0,699 ; alto entre 0,700 e 0,799 ; e muito alto, acima de 0,800$)$. O grupo de IDH muito baixo agrega quase a totalidade dos estados do Nordeste. Já o grupo de baixo IDH reúne os estados do Norte e os estados do Nordeste não incluídos no grupo anterior. O grupo de IDH médio reúne os estados do Centro Oeste e os dois estados do Sudeste com maior variação dos indicadores sociais, seja pelo perfil do próprio estado, ou por agregar muitos municípios e com muitas diferenças estruturais e demográficas. Finalmente, o grupo com alto IDH concentra os estados da Região Sul e Sudeste, evidenciando a disparida- 


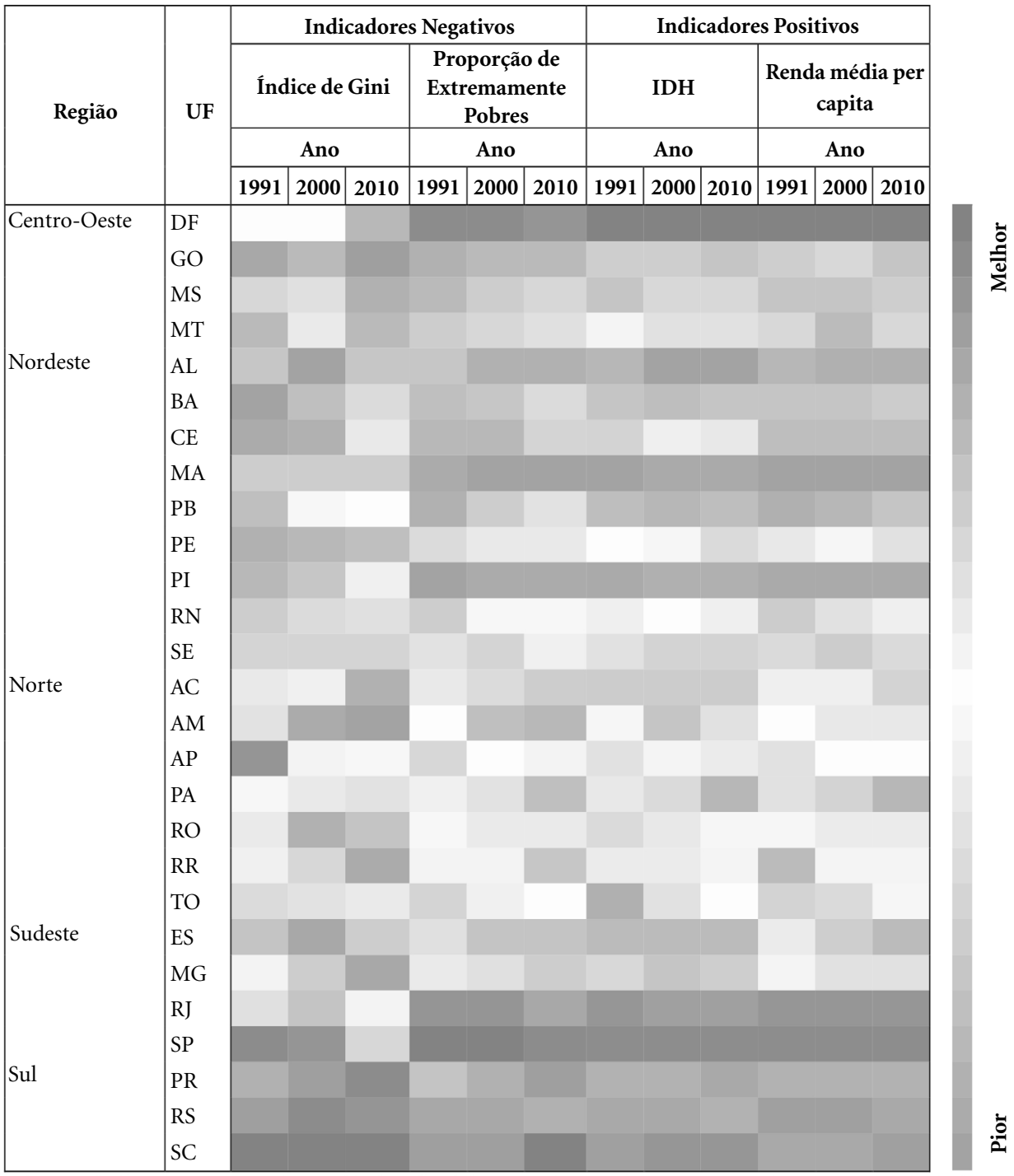

Figura 1. Ranqueamento das unidades da federação de acordo com indicadores socioeconômicos por ano censitário. Brasil, 1991-2010.

Fonte: Atlas do Desenvolvimento Humano, 2020.

de do eixo Sul-Sudeste com o Norte-Nordeste do Brasil.

Relação semelhante àquela observada para o IDH é percebida para os indicadores de renda média per capita e proporção de população extremamente pobres. Quando considerada a proporção da população extremamente pobre, houve uma notável redução desta proporção entre 1991 e 2010 e, na direção oposta, houve um incremento geral da renda média per capita. Ainda assim, a desigualdade entre Norte-Nordeste e Sul-Sudeste parece se manter, a despeito da melhora geral.
Ao analisar o indicador de desigualdade, observa-se que esta evolução ainda é acompanhada de alguma disparidade. $\mathrm{O}$ índice de Gini reduziu no Brasil em boa parte das UF. Houve, ainda, uma redução na disparidade entre as taxas das UF. Contudo, pode-se dizer que UF com maiores desigualdades têm se distanciado daquelas em que a desigualdade parece ter diminuído ou se estabilizado. É importante salientar que as mudanças nos indicadores de renda não necessariamente acompanham as tendências - em nível ou padrão - dos indicadores de desigualdade. Isto 
(a) Gini

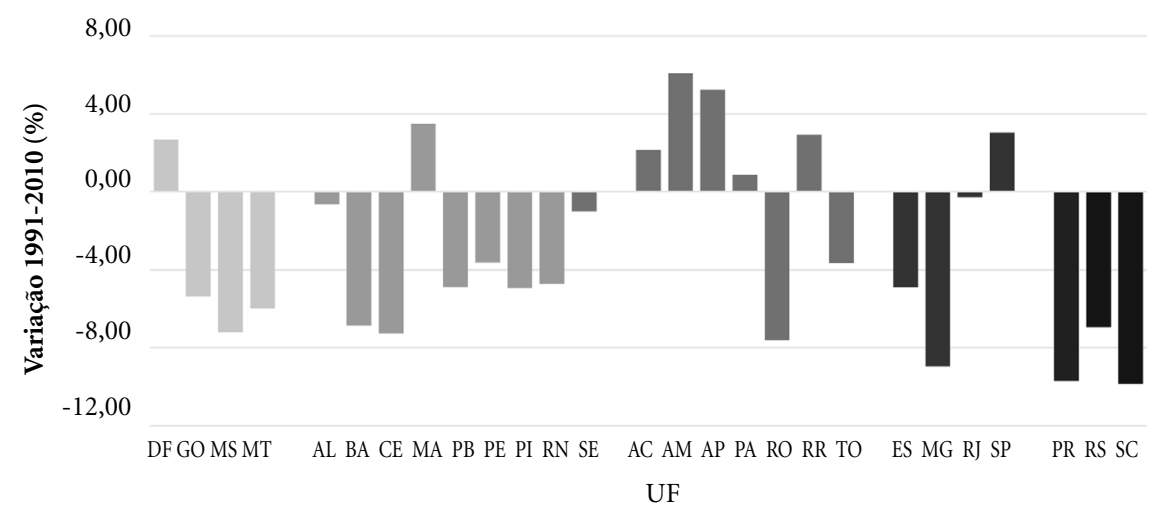

(b) IDH

100,00

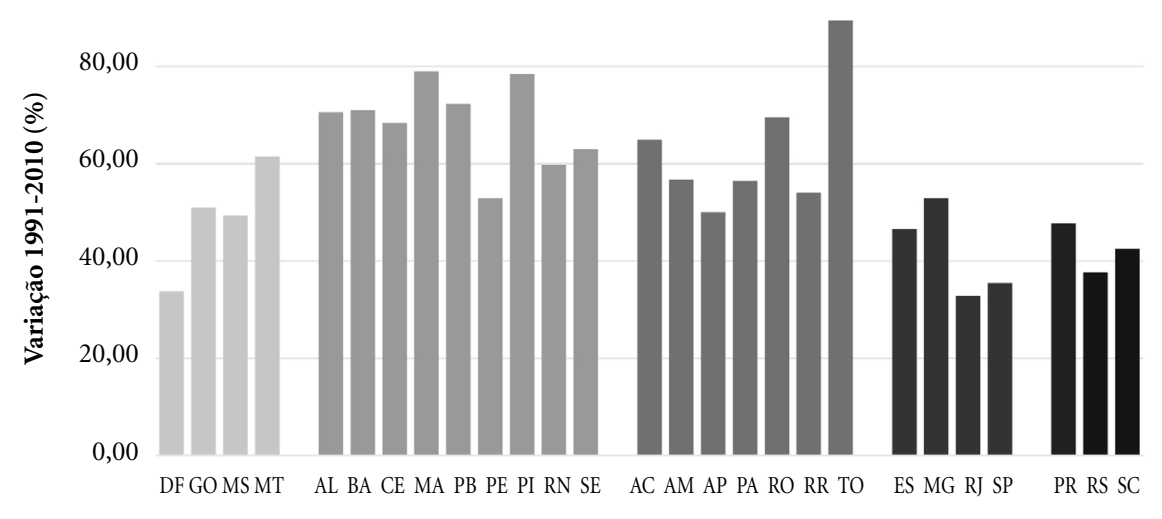

UF

continua

Figura 2. Variação relativa dos indicadores socioeconômicos por unidade da federação. Brasil, 1991-2010.

significa dizer que alguns estados possuem maior riqueza, e isto não significa necessariamente que não possuem desigualdades.

A Figura 5 destaca que há um efeito de correlação entre os indicadores econômicos e os demográficos. De uma forma geral, esta correlação é persistente no tempo. Esta evidência é mais importante para a renda média per capita e para a proporção de população em pobreza extrema, possivelmente pelo fato de estes dois indicadores não possuírem distribuição normal.

É importante observar que há uma associação entre a mortalidade e a natalidade, apresentada na Figura 6. Ao utilizar as taxas brasileiras do ano de 1991 como parâmetros de definição de alto e baixo, para comparar o comportamento destas taxas nas UF, criou-se a imagem de quatro quadrantes que, em sentido horário, descrevem quatro distintos cenários: alta natalidade e alta mortalidade; baixa mortalidade e alta natalidade; baixa natalidade e baixa mortalidade; e baixa natalidade e alta mortalidade. Estes cenários descrevem, por aproximação, as fases da transição demográfica. Nós observamos que as UF como um todo experimentam a transição ao longo dos anos (Figura 6a). Contudo, as desigualdades e a distância entre estes dois grupos persistem no tempo (Figuras 6b, 6c e 6d). Há um agrupamento espacial das UF para o estágio em que elas se encontram da transição demográfica. As unidades do Norte e do Nordeste, comparadas às unidades do Sul e Sudeste, estão sistematicamente em momentos anteriores da transição, independente do ano de observação. Quando observada 
(c) Pobreza Extrema

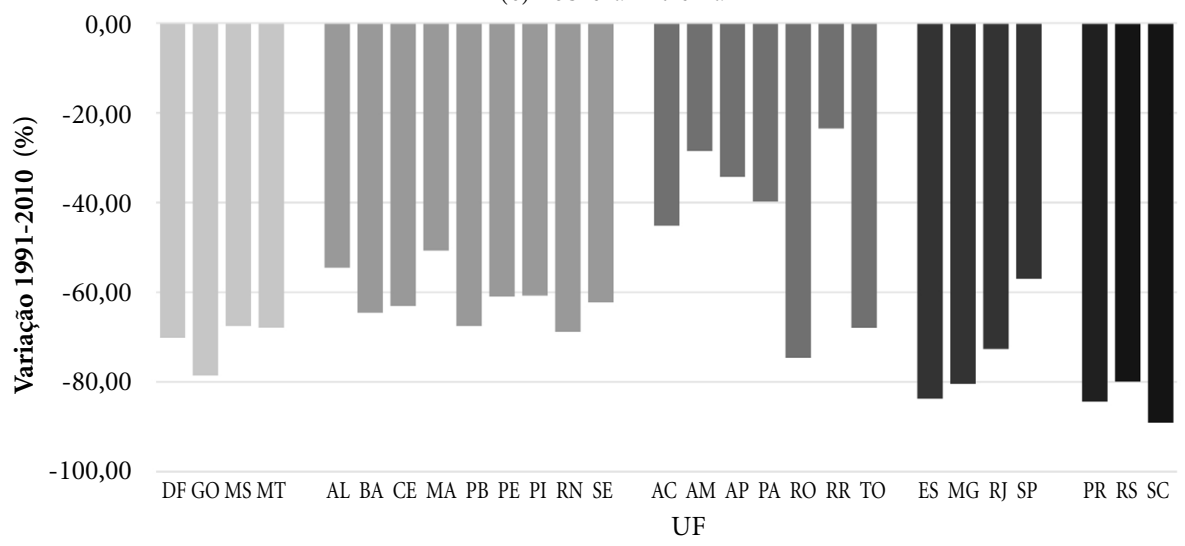

(d) Renda

160,00

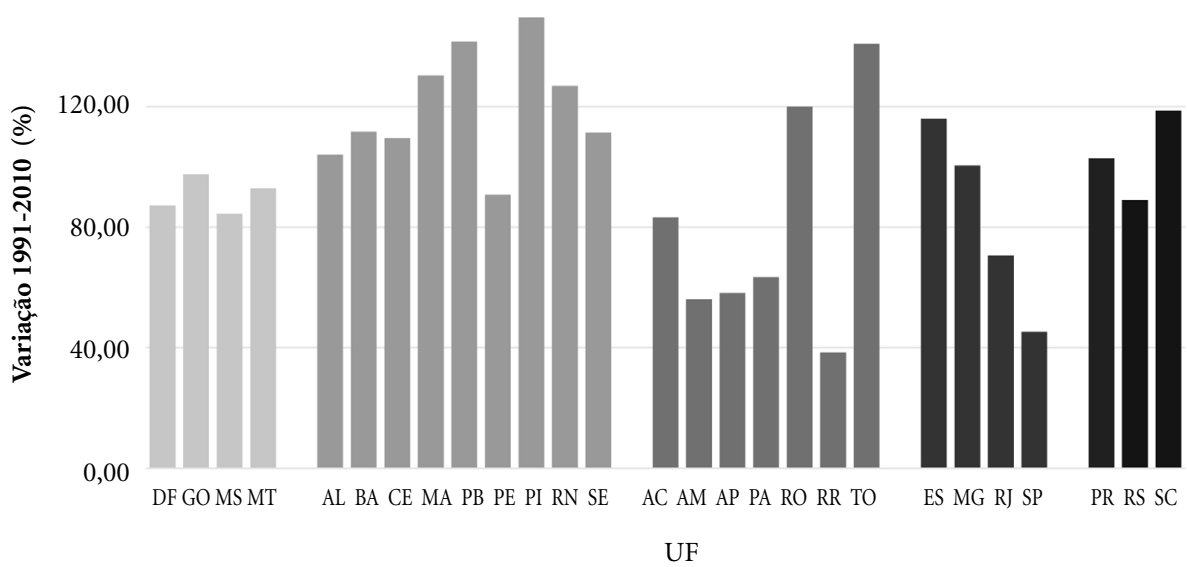

Figura 2. Variação relativa dos indicadores socioeconômicos por unidade da federação. Brasil, 1991-2010.

Fonte: Atlas do Desenvolvimento Humano, 2020.

a correlação entre a mortalidade e a natalidade (Figura 6e), nota-se que, considerando as UF dos distintos anos de análise como diferentes lugares, há uma correlação positiva entre estas duas componentes. Contudo, quando a análise é estratificada por ano censitário (Figura 6f), a correlação se torna negativa para todos os anos. Esta mudança, acredita-se, tem relação com uma mudança no curso da transição demográfica no país. Desta forma, notamos que o ano de análise é um confundidor, e ele evidencia o paradoxo de Simpson para este fenômeno.

De forma preliminar, é importante reconhecer certo padrão nos principais indicadores que descrevem as fases da transição demográfica no Brasil (Tabela 2). De uma forma geral, o Brasil mudou a relação entre natalidade e mortalidade entre 1991 e 2010. Nos anos de 1991 e 2000 a relação era direta, caracterizando uma etapa anterior da transição demográfica. Vale mencionar que a correlação era mais forte em 1991 do que em 2000, embora nos dois anos ela fosse não significativa. Já em 2010 a relação se inverte, passando a ser inversa (ainda que não possua significância estatística).

Finalmente, quando estas correlações são analisadas estratificadas pelos indicadores de desigualdade, desenvolvimento, pobreza e renda, observa-se um fenômeno atípico, ratificando a existência do Paradoxo de Simpson. Algumas destas correlações, no conjunto de dados, se mostram sem significância estatística, em uma 


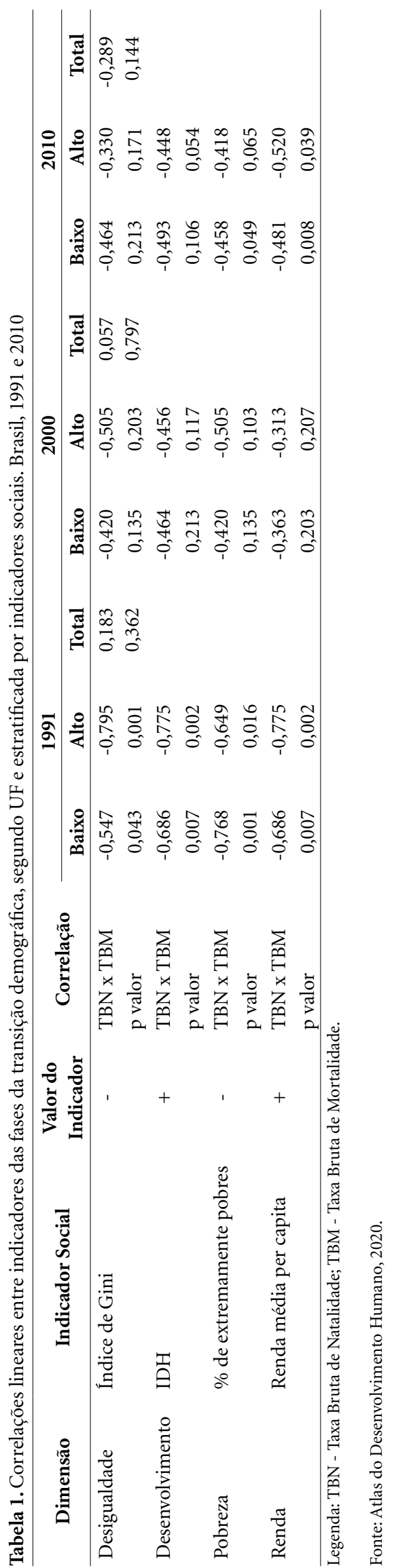

determinada direção. Quando estratificadas, passam a ter comportamento oposto ao conjunto de dados geral, e passando a apresentar significância em alguns casos. Esta situação é particularmente notada no ano de 1991, em que não só o sentido da correlação muda nos grupos, como passa a ter significância estatística. No ano de 2000, permanece a mudança do sentido da correlação, contudo sem significância estatística, sugerindo que a transição se encontra em curso, o que diminui a distância entre as UF no que diz respeito ao nível de natalidade e mortalidade. Em 2010, as correlações dos subgrupos passam a ter mesmo sentido que a correlação total, e o único indicador que reflete correlação significativa é o indicador de renda. Este fenômeno sugere que a renda, a pobreza, o desenvolvimento e a desigualdade podem se comportar como confundidores que revertem o sinal na relação entre as componentes demográficas, com alguma diferença na força de interação.

\section{Discussão}

O crescimento da população não é um fenômeno independentemente do tipo de organização social existente. Os dados analisados sugerem que há uma relação entre o nível de renda e pobreza, associados ao padrão de desigualdade social e espacial, e a dinâmica demográfica do país. Em que pese o fato de haver uma relação entre pobreza, desigualdade, e o "pace" da transição demográfica no Brasil, o que se tem é uma heterogeneidade regional expressiva no Brasil, o que parece criar dois grandes blocos (Norte/Nordeste do país; e Sul/Sudeste), que parecem caminhar em sentidos opostos, expressos tanto nos indicadores demográficos da transição quanto nos indicadores econômicos ${ }^{30}$.

A discussão central proposta pelo presente estudo se baseia em um fato crucial: a direção das setas causais é determinada pela estrutura causal do problema, não por considerações estatísticas. Assim, um tratamento do exemplo de Simpson que ignora a estrutura causal não contém informações suficientes para determinar a adequação da medida de associação marginal versus condicional $^{31}$. A assunção de que o paradoxo de Simpson se trata uma simples relação de confundimento deixa de lado o ponto principal do estudo original que o identificou: o raciocínio estatístico é insuficiente para escolher entre a medida de associação marginal e condicional ${ }^{32}$. Na verdade, há uma compreensão de que a informação estatísti- 

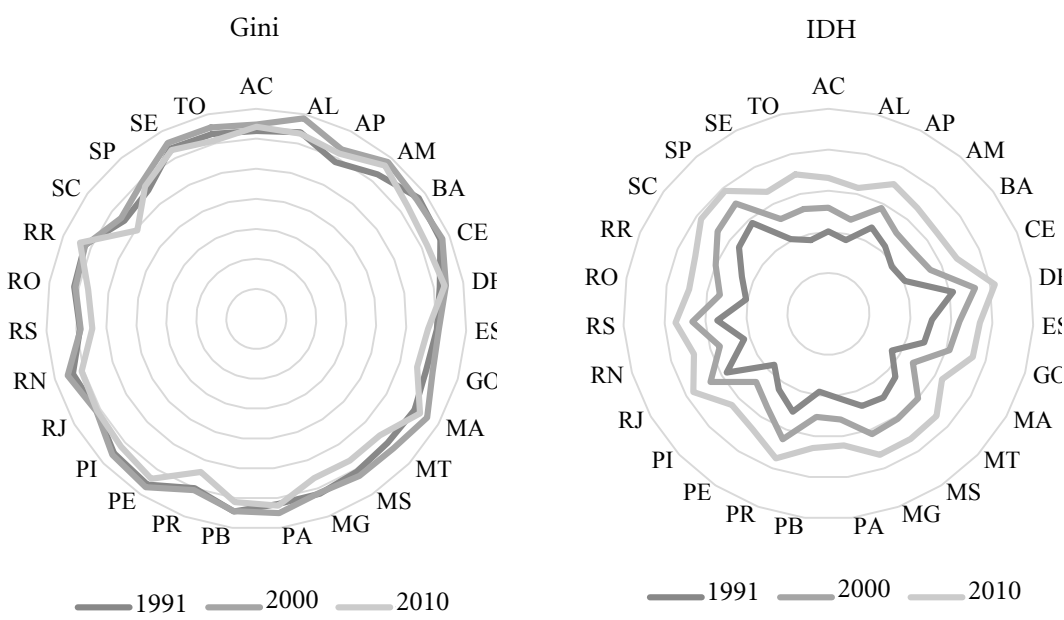

$\%$ pobres

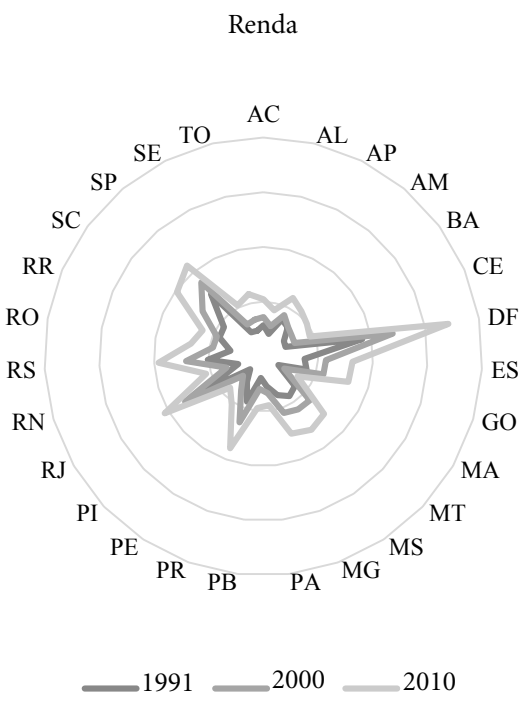

Figura 3. Evolução dos indicadores socioeconômicos segundo UF por ano censitário. Brasil, 1991-2010.

Fonte: Atlas do Desenvolvimento Humano, 2020.

ca precisa ser reforçada com um modelo teórico adequado para inferência causal a partir de dados observacionais $^{33}$.

Possivelmente, a principal razão pela qual a associação foi revertida é porque a probabilidade de nascimentos e de morte varia de acordo com os estratos sociais e a exposição à pobreza. Esta relação está documentada na literatura ${ }^{34-36}$. Isto significa assumir que a transição demográfica possui íntima relação com aspectos sociais elementares, como a divisão em classes sociais, a hierarquia social, a pobreza e a desigualdade ${ }^{37}$, e que estes elementos estão na estrutura causal dos fenômenos descritores da transição, a saber: nascimentos e mortes.

Soma-se a isso o fato de que o modelo de transição demográfica não é preditivo. De fato, como todos os modelos, o modelo de transição demográfica tem suas limitações. Por exemplo, o modelo pressupõe que, com o tempo, todos os países passem pelos mesmos quatro estágios: o primeiro, com crescimento demográfico quase nulo, graças às altas taxas de natalidade e de mortalidade; o segundo, com declínio acelerado das taxas de mortalidade, seguido de uma queda visivelmente mais lenta das taxas de natalidade; o 

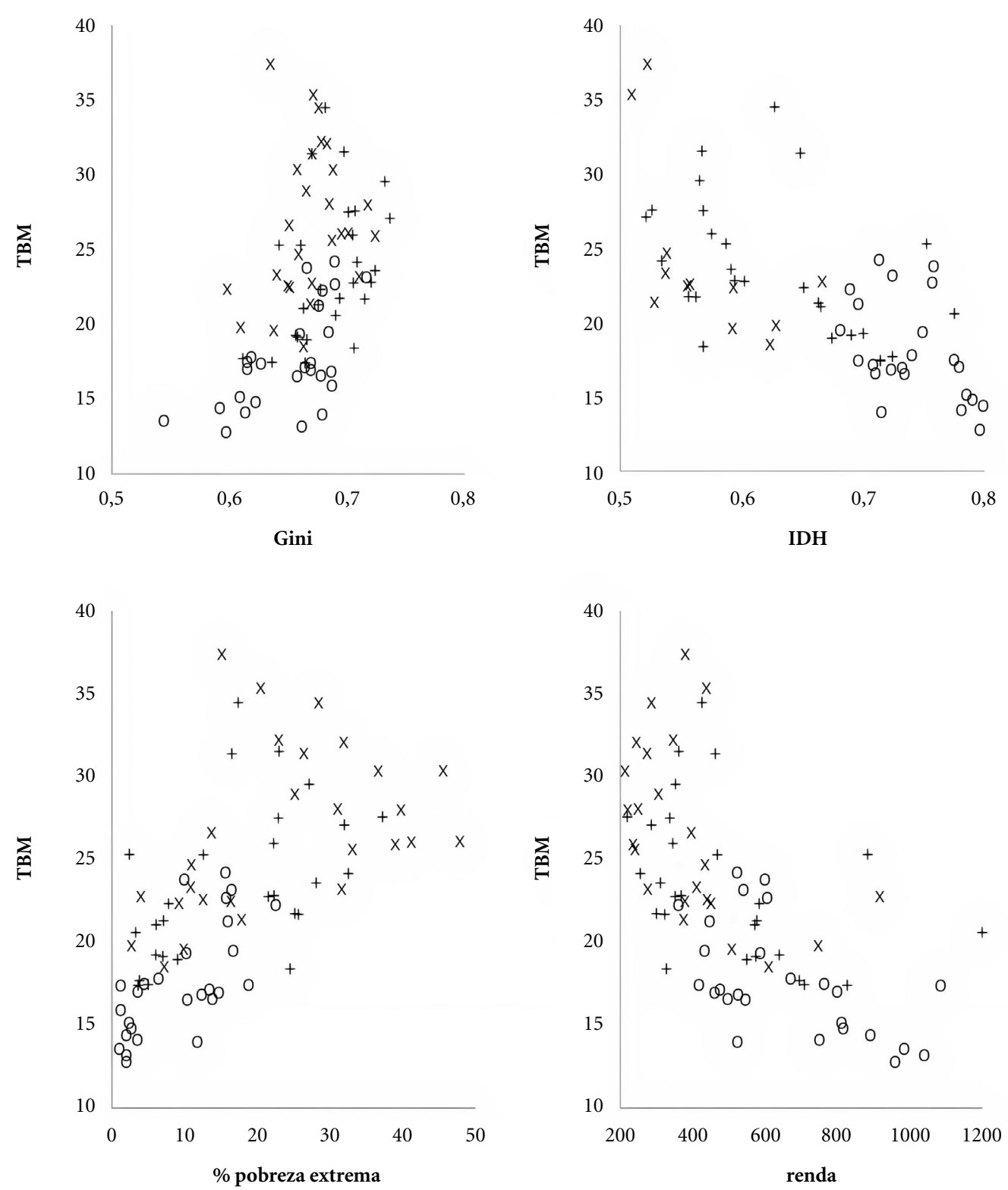

Legenda

$\times 1991$

$+2000$

O 2010

continua

Figura 4. Dispersão das taxas de natalidade e mortalidade segundo indicadores socioeconômicos por anos censitários. Brasil, 1991-2010.

terceiro, com a aceleração da queda da natalidade; e o quarto nível, com baixas taxas de natalidade e de mortalidade, apontando para um discreto aumento da mortalidade ${ }^{1,38}$.

Ao levar em consideração esta perspectiva, é importante situar que os estágios mencionados dependem de um processo contínuo de industrialização e, consequentemente, de urbanização. E este fenômeno parece ser imprevisível para alguns países de renda baixa africanos, ou para países com estagnação de crescimento criado pela periferização dos processos produtivos ${ }^{18}$. 

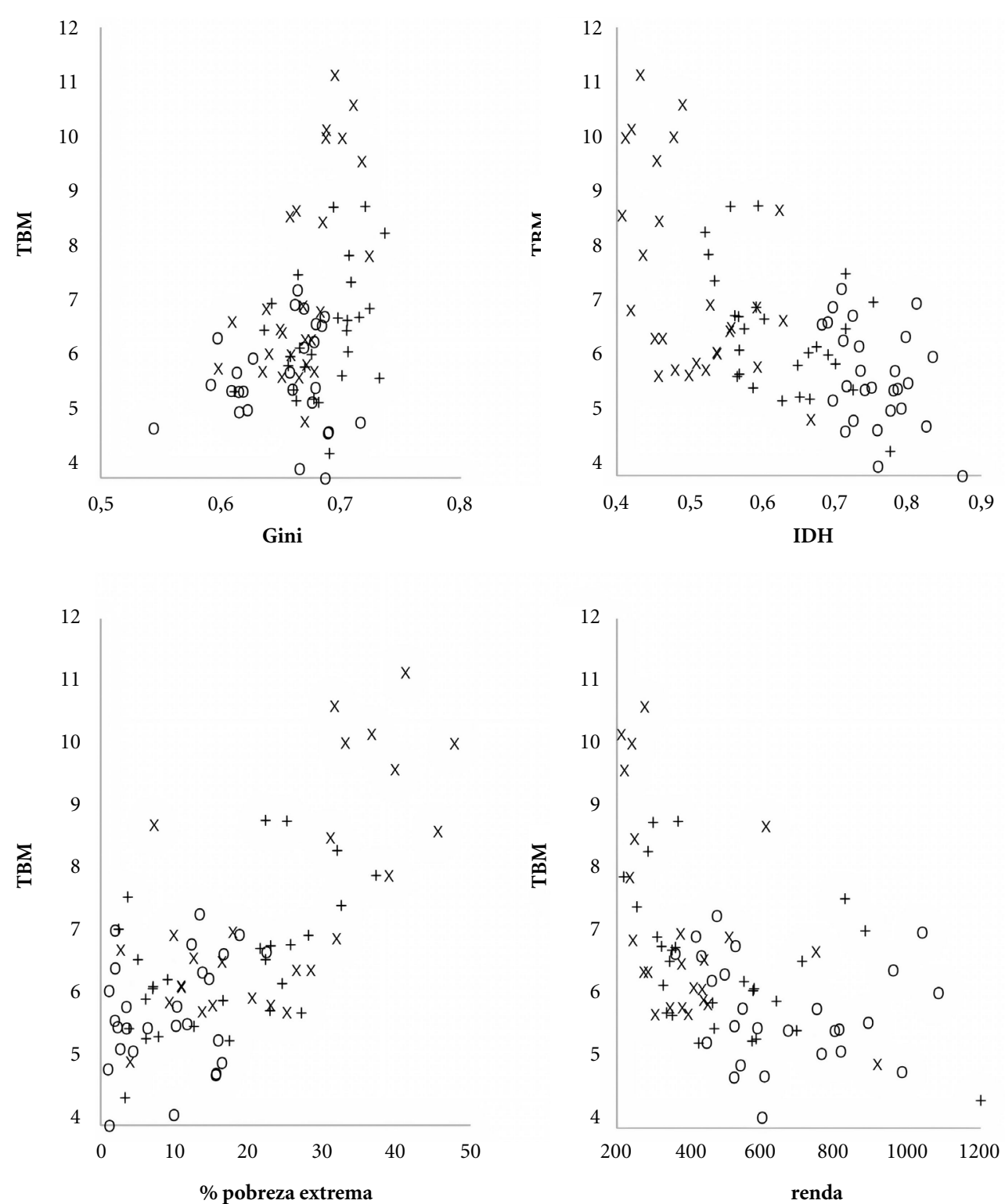

Legenda

$\times 1991$

$+2000$

O 2010

Figura 4. Dispersão das taxas de natalidade e mortalidade segundo indicadores socioeconômicos por anos censitários. Brasil, 1991-2010.

Fonte: Atlas do Desenvolvimento Humano, 2020.

Além disso, a escala de tempo do modelo, especialmente em vários países do Sudeste Asiático, é absolutamente distinta daquela observada nos países europeus, à medida que se desenvolvem a uma taxa muito mais rápida do que os primeiros países industrializados ${ }^{39}$.
Parece conveniente dizer que a relação entre desenvolvimento e transição demográfica não é unilateral: o contexto de desigualdade, pobreza e crescimento econômico influenciam os nascimentos e os óbitos e, no sentido oposto, o comportamento da natalidade e da mortalidade, uma 


\begin{tabular}{|c|c|c|c|c|c|c|c|c|c|c|c|}
\hline \multirow{2}{*}{ 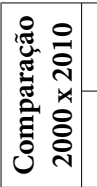 } & $\sum_{\text {鬲 }}$ & \multicolumn{2}{|c|}{$\rightarrow$} & $\leftarrow$ & \multicolumn{2}{|c|}{$\rightarrow$} & $\leftarrow$ & \multicolumn{2}{|c|}{$\rightarrow$} & \multicolumn{2}{|l|}{$\uparrow$} \\
\hline & Z & $\leftarrow$ & & $\uparrow$ & $\leftarrow$ & & $\uparrow$ & $\uparrow$ & & $\rightarrow$ & \\
\hline \multirow{6}{*}{ ڤั่ } & $\sum_{\mathscr{M}}$ & $\begin{array}{l}\hat{0} \\
0 \\
0\end{array}$ & $\begin{array}{l}\mathbb{N} \\
\hat{0}\end{array}$ & \begin{tabular}{l|l}
9 & \\
0 \\
0 \\
1
\end{tabular} & 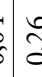 & $\frac{\partial}{0}$ & 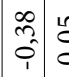 & $\begin{array}{lll}0 \\
0 \\
0\end{array}$ & $\because$ & - & \\
\hline & Z & $\begin{array}{l}\vec{b} \\
\dot{0}\end{array}$ & $\begin{array}{l}8 \\
0 \\
0\end{array}$ & 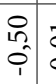 & $\hat{s}$ & $\begin{array}{l}8 \\
0 \\
0\end{array}$ & 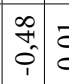 & 它 & & & 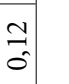 \\
\hline & 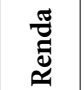 & $\begin{array}{c}\hat{m} \\
\hat{i}\end{array}$ & $\begin{array}{l}\Delta \\
0 \\
0\end{array}$ & $\begin{array}{c}\overrightarrow{1} \\
\hat{\sigma}\end{array}$ & $\begin{array}{l}\infty \\
c\end{array}$ & $\begin{array}{l}8 \\
0 \\
0\end{array}$ & - & \begin{tabular}{l}
$\infty$ \\
\multirow{1}{*}{} \\
$c$ \\
1
\end{tabular} & 0 & & $\begin{array}{l}n \\
0 \\
0 \\
0\end{array}$ \\
\hline & 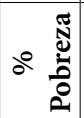 & $\begin{array}{l}\frac{1}{2} \\
\hat{0}\end{array}$ & $\begin{array}{l}8 \\
8 \\
0\end{array}$ & $\begin{array}{l}\text { o. } \\
\hat{1}\end{array}$ & - & & \begin{tabular}{|c|c}
0 & 8 \\
0 \\
0
\end{tabular} & $\begin{array}{lll}8 & \infty \\
0 & 0 \\
0\end{array}$ & 8 & & $\frac{\partial}{0}$ \\
\hline & 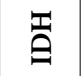 & 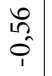 & $\begin{array}{l}8 \\
8\end{array}$ & - & $\begin{array}{l}8 \\
\text { gे } \\
1\end{array}$ & $\mid \begin{array}{l}8 \\
0 \\
0\end{array}$ & \begin{tabular}{|c|c} 
& 8 \\
0 &
\end{tabular} & $\begin{array}{lll}8 & 0 \\
0 & 0 \\
0 & \\
0\end{array}$ & $\begin{array}{l}0 \\
0 \\
0\end{array}$ & $\left|\begin{array}{c}q \\
0 \\
1 \\
1\end{array}\right|$ & $\begin{array}{l}\overrightarrow{1} \\
0 \\
0\end{array}$ \\
\hline & 菏 & $\neg$ & & \begin{tabular}{l|l}
0 \\
Ln \\
0 \\
0
\end{tabular} & 占 & $\begin{array}{l}8 \\
0 \\
0\end{array}$ & \begin{tabular}{|c|c}
$\hat{m}$ & 0 \\
$\hat{i}$ & 0 \\
1 & 0
\end{tabular} & $\begin{array}{ll}0 & 0 \\
0 & 0 \\
0 & 0\end{array}$ & 8 & & $\begin{array}{ll}N & \frac{1}{2} \\
0\end{array}$ \\
\hline \multirow{2}{*}{ 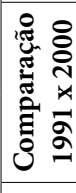 } & 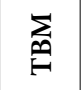 & \multicolumn{2}{|l|}{$\rightarrow$} & \multicolumn{2}{|c|}{$\uparrow$} & $\rightarrow$ & \multicolumn{2}{|l|}{$\leftarrow$} & $\leftarrow$ & \multicolumn{2}{|l|}{$\uparrow$} \\
\hline & 营 & \multicolumn{2}{|l|}{$\leftarrow$} & $\leftarrow$ & \multicolumn{2}{|c|}{$\leftarrow$} & $\leftarrow$ & \multicolumn{2}{|c|}{$\uparrow$} & \multicolumn{2}{|l|}{$\leftarrow$} \\
\hline \multirow{6}{*}{ ๕్టి } & 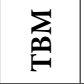 & $\begin{array}{l}q \\
q \\
0\end{array}$ & $\begin{array}{l}+ \\
0 \\
0\end{array}$ & 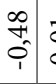 & $\tilde{n}$ & $\begin{array}{l}3 \\
0 \\
0\end{array}$ & 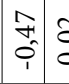 & $\begin{array}{lll}0 \\
0 \\
0\end{array}$ & o & -1 & \\
\hline & Z & $\begin{array}{l}F \\
0 \\
0\end{array}$ & $\begin{array}{l}\tilde{0} \\
0 \\
0\end{array}$ & \begin{tabular}{l|l}
0 & \\
+ \\
1 \\
1
\end{tabular} & in & $\mid \begin{array}{l}3 \\
0 \\
0\end{array}$ & 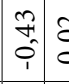 & $\begin{array}{l}0 \\
0 \\
0\end{array}$ & & $\begin{array}{l}8 \\
0 \\
0\end{array}$ & $\begin{array}{l}2 \\
\hat{\sigma}\end{array}$ \\
\hline & $\begin{array}{l}\text { 营 } \\
\widetilde{\approx}\end{array}$ & $\begin{array}{l}0 \\
0 \\
0 \\
1\end{array}$ & $\begin{array}{l}8 \\
0 \\
0\end{array}$ & $\begin{array}{l}\vec{a} \\
0 \\
0\end{array}$ & 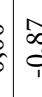 & $\begin{array}{l}8 \\
0 \\
0\end{array}$ & - & ? & $\tilde{O}$ & f & $\begin{array}{lll}\delta & = \\
0 & 0 \\
0 & 0\end{array}$ \\
\hline & 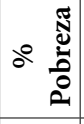 & $\begin{array}{l}+ \\
\infty \\
0 \\
0\end{array}$ & $\begin{array}{l}8 \\
0\end{array}$ & $\begin{array}{c}\text { th } \\
\hat{1}\end{array}$ & - & & \begin{tabular}{|c|c|c}
0 & 8 \\
0 & 0 \\
1 & 0
\end{tabular} & $\begin{array}{ccc}8 & 0 \\
0 & 0\end{array}$ & $\left\{\begin{array}{l}\overline{0} \\
0\end{array}\right.$ & חn & : \\
\hline & 䆩 & \begin{tabular}{|l|} 
\\
1 \\
0 \\
1 \\
\end{tabular} & $\begin{array}{l}8 \\
0 \\
0\end{array}$ & - & $\begin{array}{l}5 \\
\text { वे } \\
\text { in }\end{array}$ & $\begin{array}{l}8 \\
0 \\
0\end{array}$ & $\begin{array}{l}+ \\
\hat{\sigma}\end{array}$ & $\begin{array}{ll}0 \\
0 \\
0 \\
0\end{array}$ & $\overrightarrow{0}$ & $\mid \begin{array}{l}\infty \\
+ \\
0 \\
1 \\
1\end{array}$ & $\begin{array}{lll}0 & 0 \\
0 & 0 \\
0 & 0.0\end{array}$ \\
\hline & 节 & - & & \begin{tabular}{l|l}
$\infty$ & \\
$\hat{0}$ & \\
1 & \\
\end{tabular} & $\vec{c}$ & $\begin{array}{l}8 \\
0 \\
0\end{array}$ & \begin{tabular}{l|l}
$\hat{0}$ & 8 \\
0 & 8
\end{tabular} & $\begin{array}{lll}8 & 7 \\
0 & \\
0\end{array}$ & $\begin{array}{l}m \\
0 \\
0\end{array}$ & 辛 & $\begin{array}{ll}5 & = \\
0 & 0 \\
0 & 0\end{array}$ \\
\hline \multirow{6}{*}{ ลू } & 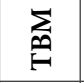 & $\begin{array}{l}\vec{b} \\
0 \\
0\end{array}$ & $\begin{array}{l}8 \\
0 \\
0\end{array}$ & \begin{tabular}{l|l}
$\infty$ & \\
+ \\
0 \\
1
\end{tabular} & 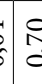 & $\begin{array}{l}8 \\
0 \\
0\end{array}$ & \begin{tabular}{|c|c}
$n$ & $g$ \\
0 & 0 \\
0 & 0
\end{tabular} & $\begin{array}{lll}0 \\
0 \\
0 \\
0\end{array}$ & 合 & -1 & $\tau$ \\
\hline & Z & $\begin{array}{l}n \\
\\
0 \\
\end{array}$ & 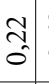 & \begin{tabular}{l|l}
0 & \\
0 \\
0
\end{tabular} & $\stackrel{9}{q}$ & $\mid \begin{array}{l}1 \\
0 \\
0 \\
0\end{array}$ & 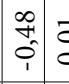 & : & & \begin{tabular}{l}
7 \\
\hdashline \\
1 \\
1
\end{tabular} & $\begin{array}{ll}\hat{n} & = \\
0 & 0 \\
0\end{array}$ \\
\hline & 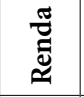 & $\begin{array}{l}n \\
n \\
0 \\
0 \\
1\end{array}$ & 8 & $\hat{\delta}$ & {$\left[\begin{array}{l}\infty \\
\infty \\
c\end{array}\right.$} & 8 & - & 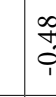 & 0 & $\begin{array}{c}n \\
1 \\
0 \\
i \\
\end{array}$ & 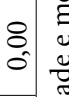 \\
\hline & 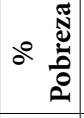 & $\begin{array}{l}n \\
\hat{n} \\
0\end{array}$ & $\begin{array}{l}8 \\
0 \\
0\end{array}$ & $\begin{array}{l}2 \\
\hat{i}\end{array}$ & - & & $\mid \begin{array}{c}0 \\
\infty \\
0 \\
1\end{array}$ & $\begin{array}{c}8 \\
0 \\
0\end{array}$ & Oे & R & $\begin{array}{l}8 \\
0 \\
0\end{array}$ \\
\hline & 票 & \begin{tabular}{|l|} 
\\
0 \\
0 \\
1 \\
\end{tabular} & $\begin{array}{l}8 \\
8 \\
0\end{array}$ & - & $\begin{array}{l}3 \\
0 \\
i\end{array}$ & $\begin{array}{l}8 \\
0 \\
0\end{array}$ & \begin{tabular}{c|c}
2 \\
$\hat{\sigma}$ & 8 \\
\end{tabular} & 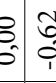 & 8 & $\begin{array}{l}0 \\
+ \\
0 \\
1 \\
1\end{array}$ & $\begin{array}{l}\overrightarrow{0} \\
0 \\
0\end{array}$ \\
\hline & 洁 & - & & $\begin{array}{l}\overrightarrow{0} \\
0 \\
0 \\
1\end{array}$ & 络 & 8 & \begin{tabular}{l|l}
$n$ \\
$n$ \\
2 & 8 \\
0 & 8 \\
1 & 0
\end{tabular} & 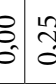 & $\hat{\tilde{c}}$ & $\mid \begin{array}{l}\overrightarrow{0} \\
0 \\
0\end{array}$ & 8 \\
\hline & & $\rightarrow$ & 2 & +1 & 5 & a & +1 & - 1. & $=$ & -4 & 2 \\
\hline & & :ंี & & $\vec{T}$ & 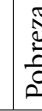 & & 苞 & $\stackrel{n}{1}$ & & $\sum_{\substack{M \\
F}}$ & \\
\hline
\end{tabular}

vez que modificam a estrutura etária da população, também influenciam a velocidade de desenvolvimento dos países?.

Ainda que haja uma intenção de que as políticas públicas mitiguem determinadas tendências de aumento das disparidades, aplacando desigualdades socioespaciais, estudo de Arretche ${ }^{40}$ destaca que, apesar de avanços em alguns indicadores, a face territorial das desigualdades ainda é marcante no Brasil. Esta marca é percebida também em estudos exclusivos do campo da saúde pública $^{41,42}$. Desta forma, é preciso formular um planejamento regional que combine incentivos econômicos e políticas sociais para a melhoria das condições de vida e redução das desigualdades.

Naturalmente, algumas das análises realizadas possuem ressalvas. Não há consenso sobre o fato de haver uma única transição demográficas, ou múltiplas. Isto significa dizer que o comportamento da dinâmica demográfica do país pode estar influenciado por outros aspectos, como as mudanças nos arranjos familiares e padrões de nupcialidade. Além disso, é necessário se apropriar de um debate a respeito do que as etapas da transição representam: ao contrário do que didaticamente se estabelece, não há cenários fixos em que as etapas de transição significam saltos entre eles. Ao contrário, há um movimento processual que deve ser avaliado com cuidado, sob risco de não considerarmos os efeitos contextuais que são mediadores e/ou estão implicados na velocidade com que os fenômenos demográficos ocorrem.

Finalmente, a proposta de ranqueamento de UF pode trazer certo dissenso como critério de classificação da etapa de desenvolvimento ou progresso social. Ressalta-se, aqui, que ela foi utilizada exclusivamente para fins ilustrativos, no sentido de demonstrar certa dinâmica diferenciada pelas UF, sugerindo haver uma heterogeneidade estrutural no Brasil, cujo conceito foi elaborado anteriormente. Isto posto, cabe utilizar este diagnóstico inicial como ponto de partida para a análise mais robusta do cenário de transição demográfica no Brasil.

$\mathrm{Na}$ presença do paradoxo de Simpson, os resultados das análises dos dados gerais contradizem as descobertas de todos os subgrupos dos mesmos dados. Métodos de análise de dados que não levam em conta o fator de confusão, incluindo análise de tabela epidemiológica $2 \times 2$, teste $t$ de amostras independentes, teste de soma de postos de Wilcoxon, teste de qui-quadrado e análise de regressão univariável, não podem gerenciar o problema do Paradoxo de Simpson e 
(a) Dispersão

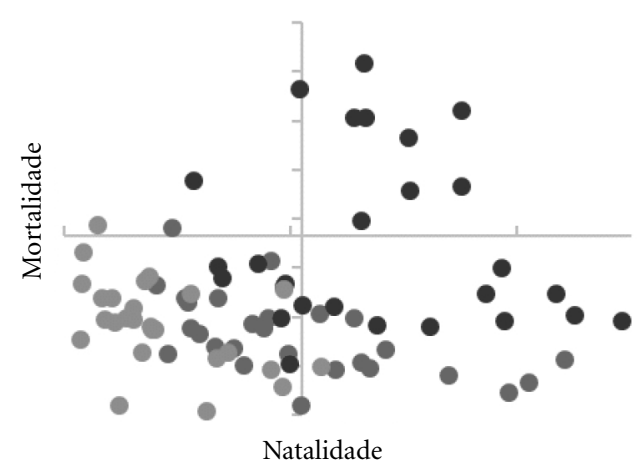

- 2000 • $1991 \quad 2010$

(c) 2000

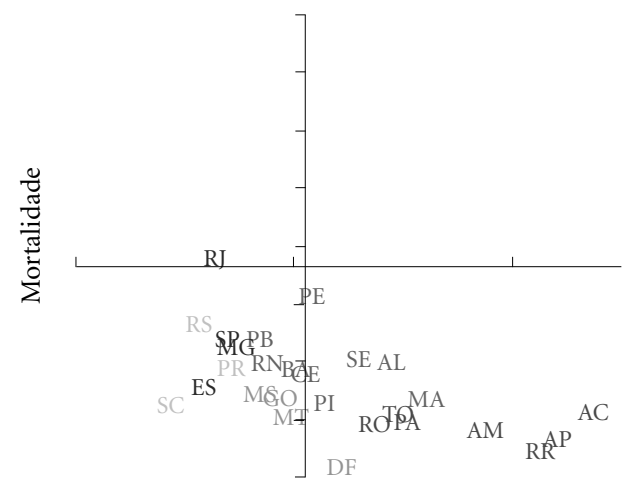

Natalidade

(e) Correção simples

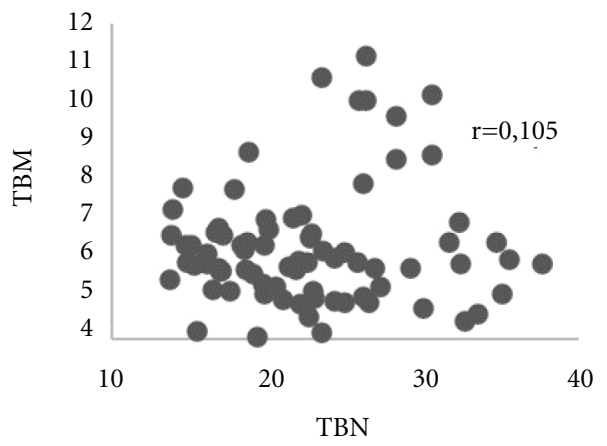

(b) 1991

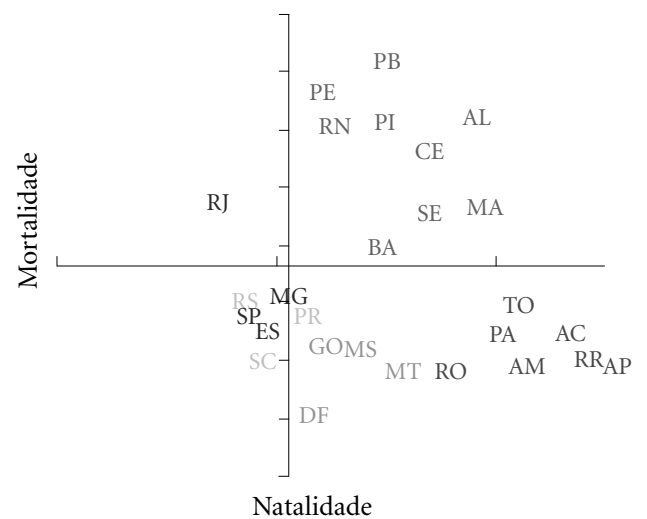

(d) 2010

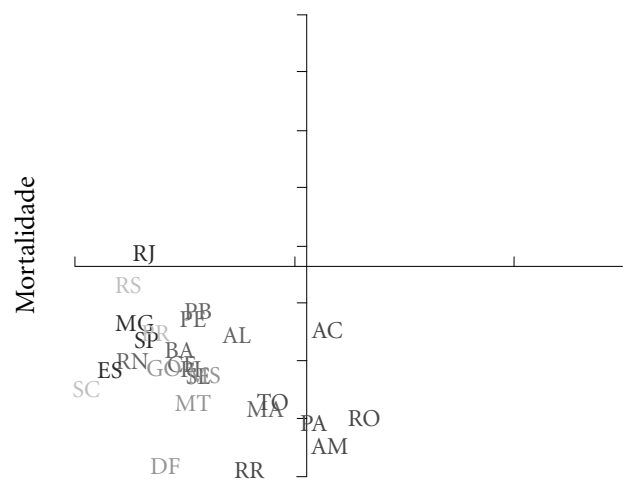

Natalidade

(f) Correlação por ano

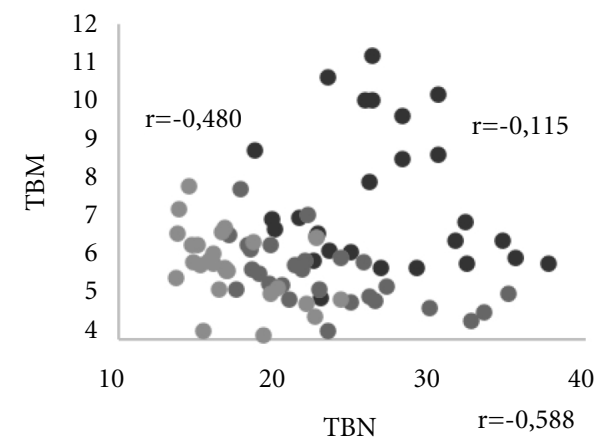

- $1991000 \quad 2010$

Legenda: Norte $\square$ Nordeste Sudeste $\square$ Centro Oeste

Figura 6. Correlação entre taxas de natalidade e mortalidade segundo UF e ano censitário. Brasil, 1991-2010.

Fonte: Atlas do Desenvolvimento Humano, 2020. 


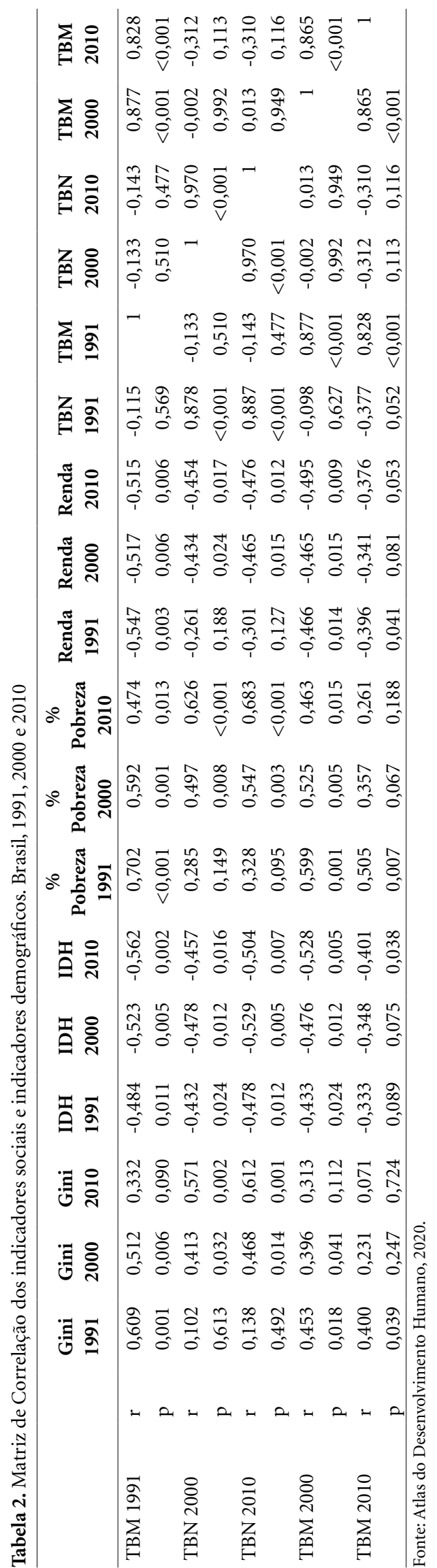

enganar as conclusões da pesquisa. $\mathrm{O}$ procedimento de Mantel-Haenszel e os métodos de regressão multivariável são exemplos de métodos de análise racionais que levam a resultados váli$\operatorname{dos}^{31,43}$. Contudo, há uma limitação ao uso deste método, que é a quantidade diminuta de pontos de análise (apenas 27 unidades da federação). Desta forma, é importante que esta questão seja revisitada com um conjunto de dados mais robusto, e com maior intervalo temporal.

Ao final, acreditamos que o presente estudo seja um diagnóstico inicial a respeito do fenômeno estudado. Oportunamente, recomendamos que estudos subsequentes, que disponham de mais dados, possam realizar análise em painel, e com outra escala geográfica, quando possível.

\section{Colaboradores}

Todos os autores contribuíram com a concepção do estudo, coleta e análise de dados, redação e revisão crítica do manuscrito.

\section{Fonte de financiamento}

Programa Institucional de Internacionalização da Coordenação de Aperfeiçoamento de Pessoal de Nível Superior (CAPES-PRINT). 


\section{Referências}

1. Caldwell JC, Caldwell BK; Caldwell P; McDonald PF, Schindlmayr T. Demographic Transition Theory. Dordrecht, The Netherlands: Springer; 2006. p. 239.

2. Rigotti JIR. Transição Demográfica. Educ Real 2012; 37(2):467-490.

3. Lesthaeghe R. The "second demographic transition": a conceptual map for the understanding of late modern demographic developments in fertility and family formation. Historical Social Res 2011; 36(2):179-218.

4. Marmot M, Allen JJ. Social determinants of health equity. Am J Public Health 2014; 104(Supl. 4):S517-S519.

5. Link BG, Phelan JC, Miech R, Westin EL. The resources that matter: fundamental social causes of health disparities and the challenge of intelligence. $J$ Health Soc Behav 2008;49(1):72-91.

6. PatekAJ. The Malthusian theory. WMJ2006;105(2):76.

7. Bongaarts J. Human population growth and the demographic transition. Philos Trans R Soc Lond B Biol Sci 2009; 364(1532):2985-2990.

8. Galor O. The Demographic Transition: Causes and Consequences. Cliometrica (Berl) 2012; 6(1):1-28.

9. Attanasio OP, Kitao S, Violante GL. Quantifying the Effects of the Demographic Transition in Developing Economies. The B.E. J Macroecon 2006; 6(1):1-44.

10. Ranganathan S, Swain RB, Sumpter DJT. The demographic transition and economic growth: implications for development policy. Palgrave Commun 2015; $1: 15033$.

11. Carvalho JAM, Rodríguez-Wong LL. A transição da estrutura etária da população brasileira na primeira metade do século XXI. Cad Saude Publica 2008; 24(3):597-605.

12. Georges R, Maia K. A distância que nos une: um retrato das desigualdades brasileiras. Oxfam Brasil; 2017. [Acessado 2018 out 02]. Disponível em https:// www.oxfam.org.br/sites/default/files/arquivos/Relatorio_A_distancia_que_nos_une.pdf

13. Guimarães RM. Os impactos das políticas de austeridade nas condições de saúde dos países com algum tipo de crise. Trab Educ Saude 2018; 16(1):383-385.

14. Kalemli-Ozcan S. A stochastic model of mortality, fertility, and human capital investment. J Develop Economics 2003; 70(1):103-118.

15. Lehmijoki U. Demographic Transition and Economic Growth. Research Reports Kansantaloustieteen laitoksen tutkimuksia. n. 99. Finland, 2003. [cited 2020 Oct 02]. Available from: https://core.ac.uk/download/ pdf/14917275.pdf.

16. Ye L, Zhang X, Geng J. Demographic transition and economic growth: Evidence from China and United States. Int J Health Plann Manage 2020 Jan;35(1):e1-e11

17. Al-Hadithi TS, Shabila NR, Al-Tawil NG, Othman SM. Demographic transition and potential for development: the case of Iraqi Kurdistan. East Mediterr Health J 2010;16(10):1098-1102.

18. Salvati L, Carlucci M, Serra P, Zambon I. Demographic Transitions and Socioeconomic Development in Italy, 1862-2009: A Brief Overview. Sustainability, MDPI. Open Access J 2019; 11(1):1-12.
19. Baudelle G, Olivier D. Changement global, mondialisation et modèle de transition démographique: réflexion sur une exception française parmi les pays développés. Historiens et Géographes 2006; 98:177-204

20. Rojanaworarit C. Misleading Epidemiological and Statistical Evidence in the Presence of Simpson's Paradox: An Illustrative Study Using Simulated Scenarios of Observational Study Designs. J Med Life 2020; 13(1):37-44.

21. Julious SA, Mullee MA. Confounding and Simpson's Paradox. BMJ 1994; 309:1480.

22. Lijphart A. Patterns of democracy: government forms and performance in thirty-six countries. New Haven: Yale University Press; 1999.

23. Instituto de Pesquisa Econômica Aplicada (IPEA). Desenvolvimento Regional no Brasil: politicas, estratégias e perspectivas. Rio de Janeiro: IPEA; 2017.

24. United Nations Development Programme. Human Development Indices and Indicators 2018 Statistical Update. New York. [cited 2019 Sep 20]. Available from: http://hdr.undp.org/sites/default/files/2018_ human_development_statistical_update.pdf.

25. Lima EEC, Queiroz BL, Zeman K. Completeness of birth registration in Brazil: an overview of methods and data sources. Genus 2018;74:1-20.

26. Lima EEC, Queiroz BL, Zeman K. Completeness of birth registration in Brazil: an overview of methods and data sources. Genus 2018; 74:1-20.

27. Hill K, You DZ, Choi YJ. Death distribution methods for estimating adult mortality: sensitivity analysis with simulated data errors. Demogr Res 2009; 21:23554.

28. Brass W, Coale AJ. Methods of analysis and estimation. In: Brass W, Coale AJ, Demeny P, Heisel DF, editors. The Demography of Tropical Africa. Princeton NJ: Princeton University Press; 1968. p. 88-139.

29. Coale AJ, Trusell TJ. Estimating the time to which Brass estimates apply. In: Preston SH, Palloni A. Five tuning Brass type mortality estimates with data on ages of surviving children. Popul Bull United Nations 1978; 10:49-54.

30. Figueiredo E, Porto Júnior S. Persistência das desigualdades regionais no Brasil: polarização e divergência. Nova Econ 2015; 25(1):195-208.

31. Hernán MA, Clayton D, Keiding N. The Simpson's paradox unraveled. Int J Epidemiol 2011; 40(3):780-785.

32. Greenland S. Interpretation and choice of effect measures in epidemiologic analyses. Am J Epidemiol 1987; 125:761-768.

33. Marang-nav de Mheen PJ, Shojania KG. Simpson's paradox: how performance measurement can fail even with perfect risk adjustment. BMJ Qual Saf 2014; 23:701-705.

34. Vasconcelos AMN, Gomes MMF. Transição demográfica: a experiência brasileira. Epidemio Serv Saude 2012; $21(4): 10$.

35. Brito F. Transição demográfica e desigualdades sociais no Brasil. Rev Bras Estud Popul 2008; 25(1):5-26. 
36. Miranda GMD, Mendes ACG, Silva ALA. Desafios das políticas públicas no cenário de transição demográfica e mudanças sociais no Brasil. Interface (Botucatu) 2017; 21(61):309-320.

37. Januzzi PM. Pobreza, desigualdade e mudança social: trajetória no Brasil recente (1992 a 2014). Rev Estud Pesq Americas 2016; 10(3):1-29.

38. Paiva PTA, Wajnman S. Das causas às conseqüências econômicas da transição demográfica no Brasil. Rev Bras Estud Popul 2005;22(2): 303-322.

39. Megere MN, Kumar GM. Demographic Transition and Economic Development in Karnataka. J Quant Economics 2018; 16(1):173-183.

40. Arretche M. Trajetórias da desigualdade: como o Brasil mudou nos últimos 50 anos. São Paulo: Editora Unesp; 2015.

41. Viacava F, Porto SM, Carvalho CC, Bellido JG. Desigualdades regionais e sociais em saúde segundo inquéritos domiciliares (Brasil, 1998-2013). Cien Saude Colet 2019; 24(7):2745-2760.

42. Albuquerque MV, ALD Viana, Lima LD, Ferreira MP, Fusaro ER, Iozzi FL. Desigualdades regionais na saúde: mudanças observadas no Brasil de 2000 a 2016. Cien Saude Colet 2017; 22(4):1055-1064.

43. Pearl J. Understanding Simpson's Paradox. Tech. Rep. R-414 December 2013. Department of Computer Science, University of California, Los Angeles, CA. [cited 2020 Dez 20]. Available from: em https://ftp. cs.ucla.edu/pub/stat_ser/r414.pdf

Artigo apresentado em 20/11/2020

Aprovado em 20/05/2021

Versão final apresentada em 22/05/2021

Editores-chefes: Romeu Gomes, Antônio Augusto Moura da Silva 
